\title{
Alteration of the multi-segment foot motion during gait in individuals with plantar fasciitis: a matched case-control study
}

\author{
SunEe BOVONSUNTHONCHAI ${ }^{1}$, SUTHASINEE THONG-ON ${ }^{1}$, ROONGTIWA VACHALATHITI ${ }^{1}$, \\ WARINDA INTIRAVORANONT ${ }^{2}$, SARAWUT SUWANNARAT ${ }^{2}$, RICHARD SMITH ${ }^{3}$ \\ ${ }^{1}$ Faculty of Physical Therapy, Mahidol University, Nakhon Pathom, Thailand. \\ ${ }^{2}$ Physical Therapy Center, Faculty of Physical Therapy, Mahidol University, Bangkok, Thailand. \\ ${ }^{3}$ Discipline of Exercise and Sport Science, Faculty of Health Science, The University of Sydney, Australia.
}

\begin{abstract}
Purpose: The objective of this study was to compare the ground reaction forces (GRFs) and the multi-segment foot motion between individuals with plantar fasciitis (PF) and healthy controls. Methods: Twenty-one individuals with PF and 21 matched-case healthy controls who passed the criteria participated in the study. Gait data were assessed during their self-selected comfortable speeds by the 3D motion analysis system. The multi-segment foot motions were determined by the Oxford Foot Model. Outcome measures included the vertical and antero-posterior ground reaction forces (GRFs) and the multi-segment foot motions [the dorsiflexion (DF), plantarflexion $(\mathrm{PF})$, inversion (Inv), eversion (Eve), adduction (Add), and abduction (Abd) peak angles for the forefoot with respect to hindfoot (FFHF) and the DF, PF, Inv, Eve, internal rotation (IR), and external rotation (ER) peak angles for the hindfoot with respect to tibia (HFTB) as well as their ranges (R)]. Results: Comparisons between individuals with PF and healthy controls showed no significant differences in any of the GRFs. Significant reductions were found in the FFHF-DF, FFHF-DF-R, FFHF-Inv, and HFTB-Inv/Eve-R in individuals with PF. In addition, there were tendencies of the increased angles of the FFHF-PF, HFTB-DF, HFTB-Inv, and HFTB-ER, but not significantly for individuals with PF, compared to healthy controls. Conclusions: Adaptations of the intra-foot motion showed the reduction of some angles but no change for the GRFs in individuals with PF compared to the healthy controls when both groups walked at a similar gait speed.
\end{abstract}

Key words: gait, multi-segment foot motion, plantar fasciitis

\section{Introduction}

Plantar fasciitis (PF), is one of the foot pathologies that can cause difficulty, activities limitation, discomfort, and disability [2]. The common clinical manifestation that indicates this disease is pain under the heel, especially at the calcaneal origin of the plantar fascia. The symptom leads to individuals being unable to perform weight-bearing activities such as standing, walking, or running for a prolonged period [2]. Individuals with PF usually complain about heel pain at the first step in the morning or after a prolonged period of resting, as well as having sharp pain when palpating at the medial plantar calcaneal area. This heel pain may disappear after rest but it can appear again after performing excessive activities.

The PF can occur in either athletic or non-athletic populations and both sexes, but frequently present in the active workers aged between 25 and 65 years [5], [20]. The prevalence study of foot and ankle diseases in 784 multiethnic community-dwelling older adults aged 65 or more years reported that $7 \%$ of people have pain under the heel [6]. Individuals who develop the symptom into

\footnotetext{
* Corresponding author: Sunee Bovonsunthonchai, Faculty of Physical Therapy, Mahidol University, Nakhon Pathom, 73170, Thailand. E-mail: sunee.bov@mahidol.edu

Received: July 27th, 2019

Accepted for publication: October 10th, 2019
} 
the chronic stage, usually have restriction in physical activities, social participation, and quality of life [10].

The PF is caused by excessive strain, repetitive microtrauma, or inflammation of the plantar fascia [25]. It involves several risk factors such as foot alignment, intrinsic and extrinsic foot muscle strength and endurance, obesity, increasing age, poor footwear, high physical activity level or excessive duration of weight-bearing activity, and overtraining, [2], [10], [19]. However, the evidence of these risk factors' relationship in PF is insufficient, as it is based on very few studies, small sample size, and controversial findings [15], [25].

The anatomical and biomechanical factors associated with PF is still unclear, although they are thought to be the crucial factors causing the symptom. According to the changes of these factors, as reported in previous studies, it was possible that $\mathrm{PF}$ is associated with flattening of the medial longitudinal arch [4], [18], changing of foot alignment, and reduction of ankle, subtalar and metatarsophalangeal joint range of motions [2], [10], [19]. Currently, only one study investigated the multi-segment foot motion in individuals with PF [4]. There are a number of studies about ground reaction forces (GRFs) [11], [13], [28]. However, there were controversial findings of the GRFs. Some studies reported the vertical GRF was unchanged in individuals with PF during gait [13], [28], while another reported a change in the peak magnitude [11]. This difference may result in a lack of control of gait speed during testing because individuals with PF usually walk with slow speed to prevent pain aggravation. It is known that gait speed can affect the motion and GRFs [27]. So, it is interesting to investigate the actual biomechanical changes of foot motion under the natural walking condition with the similar walking speed of individuals with PF, and matched healthy controls.

The aim of the present study was to investigate the multi-segment foot motion and GRFs in individuals with PF, compared to the healthy controls. We hypothesized that there would be differences in the multi-foot motions and GRFs between individuals with PF compared to the healthy controls.

\section{Materials and methods}

This study was a matched case-control design which was conducted from June 2017 to August 2018. The study protocol was approved by the institutional research committee board (COA no: MU-CIRB 2016/173.3012), all participants were informed about the study details and gave their informed consent before participating in the study.

\subsection{Participants}

Individuals with $\mathrm{PF}$ were recruited into the study from the Physical Therapy Center, Faculty of Physical Therapy, Mahidol University. They were screened in accordance with the study criteria by physical therapists with more than 5 years of experience in the musculoskeletal field. The inclusion criteria included: aged between $20-80$ years, a history of PF at least 1 month before enrollment, experienced pain or tenderness at the medial calcaneal tubercle or on the proximal part of plantar fascia, at least one of the clinical complaints (pain during the early steps in morning or after prolonged sitting, pain during performed a prolonged weight-bearing activities such as standing, walking, or running). They were excluded if they presented any coexisting painful conditions at the parts of lower extremity or back regions, a history of rheumatoid arthritis, neurological or vascular disease, leg length discrepancy more than $1 \mathrm{~cm}$, severe pain and unable to walk. As far as the healthy controls are concerned, they were included if they had no past or present history of the PF symptoms and no foot pain. The criteria for exclusion were the same as individuals with PF.

Forty-five individuals with PF passed the screening criteria. Due to gait requiring a well-coordinated control of both lower limbs' movement, biomechanical variables of gait may vary between those with unilateral or bilateral symptoms. Only 21 individuals with unilateral $\mathrm{PF}$ were selected to compare the multi-segment foot motions, classified into the data of symptomatic and asymptomatic sides. According to the gender, age, weight, and height could affect the biomechanical gait variables, so, these factors were matched between individuals with PF and the healthy controls.

\subsection{Data collection}

The motion capture system was calibrated prior to the data collection process. Participants changed their clothes to the close-fitting and sleeveless shirt and short pants. The anthropometric data including the height, weight, leg length, and the widths of knee and ankle were obtained to calculate the joint centres for each participant. Then, 42 retro-reflective markers with $9 \mathrm{~mm}$ in diameter were attached on the pelvis and both sides 
of the lower extremities and feet following the Oxford Foot Model (OFM) in combination with the Plug-In-Gait (PIG) [30] to obtain the multi-segment foot motion and gait parameters by an experienced investigator. To create the foot segments, markers were placed on the most distal, medial aspect of the first metatarsal shaft, the most proximal and distal lateral aspects of the fifth metatarsal shaft, and midway between second and third metatarsal heads to define the forefoot segment. For the rearfoot segment, markers were placed on sustentaculum tali, lateral calcaneus, heel (distal part of the calcaneus), posterior proximal calcaneus, and a peg marker was placed on posterior calcaneus between heel and proximal calcaneus markers. For the tibial segment, markers were placed on medial malleolus, lateral malleolus, anterior aspect of tibial crest, tibial tuberosity, and head of fibula. Prior to the data testing, a static standing still trial was captured for each participant. After that, the 6 markers (first metatarsal shaft, proximal posterior calcaneus, and medial malleolus from both sides) were removed after the static subject calibration trial was completed [24].

The OFM was developed and validated by the Nuffield Orthopeadic Centre in collaboration with Oxford University [3], [24]. It was modified and proved to be applicable with good reliability for healthy and deformed conditions both in the children and adults [24], [30]. The repeatability of OFM markers placement from our experienced investigator was performed prior to the study begin. The test-retest reliability was assessed in 10 healthy young adults over their comfortable gait speed and demonstrated good to excellent reliability $\left(\mathrm{ICC}_{3,1}\right.$ of $\left.0.738-0.922\right)$ for the FFHF and HFTB motions.

Ten high-speed infrared cameras (Vicon ${ }^{\mathrm{TM}}$, Vantage V5 series, Oxford, UK) synchronized with the 2 force plates (AMTI, model OR6-7, USA) with sampling rates at $100 \mathrm{~Hz}$ for motion and $1000 \mathrm{~Hz}$ for force data. Data were recorded during standing for $3 \mathrm{sec}$ as a static subject calibration and during walking trials over the 8-m walkway. Participants practised their walking for 2-3 trials to familiarize themselves with the environment and laboratory setting prior to collecting the real data. To prevent causing additional pain from the testing in individuals with PF, the data were collected for 3-5 successful gait trials at the individual's natural comfortable speed. Due to the effect of gait speed on the other kinematic and kinetic data, 2 trials of gait that represented a similar speed and cadence to the matched-pairs healthy controls were selected for further analyses. The averaged data from these 2 selected trials were used in the comparisons.

\subsection{Data processing}

The kinematic data was tracked using the Nexus software (version 2.8.1). The kinematic and kinetic data were filtered by the $4^{\text {th }}$ order zero-lag, low-pass Butterworth technique at cut off frequencies of $6 \mathrm{~Hz}$ and $30 \mathrm{~Hz}$, respectively. The cut off frequency for the data was tested by the residual analysis with the following formula [29];

$$
F\left(f_{c}\right)=\sqrt{\frac{1}{N} \sum_{i=1}^{N} \hat{X}_{\boldsymbol{l}}-(X i)^{2}},
$$

where:

$f_{c}=$ the cutoff frequency of the fourth-order dual-pass filter,

$X_{i}=$ raw data at $i$-th sample,

$\hat{X}_{\imath}=$ filtered data at the $i$-th sample using a fourth-order zero-lag filter.

Temporo-spatial gait parameters and kinematic data were collected from two successful trials. Initial contact and toe-off events for each foot were identified from the force plate record. The kinematic and kinetic data were normalized over a gait cycle by using MATLAB software (released R2013a, USA, license number 891627).

\subsection{Outcome measures}

The outcome measures of the study composed of the GRFs (vertical force during propulsion phase, braking, and propulsive forces $[\mathrm{N} / \mathrm{kg}]$ ) and the peak angles of the multi-segment foot motions (degrees) in three planes of movement as well as their ranges (the maximum to minimum values). The full and abbreviated names of the multi-segment foot motions are addressed in Table 1.

Table 1. The Multi-segment foot motion angles of the study

\begin{tabular}{|l|l|}
\hline \multicolumn{1}{|c|}{ Abbreviation } & \multicolumn{1}{c|}{ Full name } \\
\hline FFHF-DF & Forefoot with respect to hindfoot dorsiflexion \\
\hline FFHF-PF & $\begin{array}{l}\text { Forefoot with respect to hindfoot } \\
\text { plantarflexion }\end{array}$ \\
\hline FFHF-DF/PF-R & $\begin{array}{l}\text { Range of forefoot with respect to hindfoot } \\
\text { dorsiflexion / plantarflexion }\end{array}$ \\
\hline FFHF-Inv & Forefoot with respect to hindfoot inversion \\
\hline FFHF-Eve & Forefoot with respect to hindfoot eversion \\
\hline FFHF-Inv/Eve-R & $\begin{array}{l}\text { Range of forefoot with respect to hindfoot } \\
\text { inversion/eversion }\end{array}$ \\
\hline FFHF-Add & Forefoot with respect to hindfoot adduction \\
\hline
\end{tabular}




\begin{tabular}{|l|l|}
\hline \multicolumn{1}{|c|}{$\mathbf{1}$} & \multicolumn{1}{c|}{$\mathbf{2}$} \\
\hline FFHF-Abd & Forefoot with respect to hindfoot abduction \\
\hline FFHF-Add/Abd-R & $\begin{array}{l}\text { Range of forefoot with respect to hindfoot } \\
\text { adduction/abduction }\end{array}$ \\
\hline HFTB-DF & Hindfoot with respect to tibia dorsiflexion \\
\hline HFTB-PF & Hindfoot with respect to tibia plantarflexion \\
\hline HFTB-DF/PF-R & $\begin{array}{l}\text { Range of hindfoot with respect to tibia } \\
\text { dorsiflexion/plantarflexion }\end{array}$ \\
\hline HFTB-Inv & Hindfoot with respect to tibia inversion \\
\hline HFTB-Eve & Hindfoot with respect to tibia eversion \\
\hline HFTB-Inv/Eve-R & $\begin{array}{l}\text { Range of hindfoot with respect to tibia } \\
\text { inversion/eversion }\end{array}$ \\
\hline HFTB-IR & $\begin{array}{l}\text { Hindfoot with respect to tibia internal } \\
\text { rotation }\end{array}$ \\
\hline HFTB-ER & $\begin{array}{l}\text { Hindfoot with respect to tibia external } \\
\text { rotation }\end{array}$ \\
\hline HFTB-IR/ER-R & $\begin{array}{l}\text { Range of hindfoot with respect to tibia } \\
\text { internal rotation/external rotation }\end{array}$ \\
\hline
\end{tabular}

\subsection{Statistical analysis}

All statistical data analyses were performed using IBM SPSS software version 20 with the $p$-value threshold set at 0.05 . Data distribution was analyzed by the Kolmogorov-Smirnov Goodness-of-Fit test. Demographic data between patients with PF and healthy controls were tested by the independent sample $t$-test, Chi-squared test, and Fisher Exact test, according to the scale type. Comparisons of the temporo-spatial and the multi-segment foot motion variables among symptomatic and asymptomatic sides of the individuals with $\mathrm{PF}$ and the data from healthy controls were tested by one-way analysis of variance (ANOVA). If the comparison data showed the $p$-value $<0.05$, this led to the potential of confidence to reject the null hypothesis. Then, the least significant difference (LSD) post-hoc analysis was used to quantify the pair of difference.

\subsection{Sample size estimation}

The required sample size of 20 per group was calculated based on our own pilot data with the comparisons of the FFHF-R angle between the symptomatic side of individuals with PF $(n=10)$ and the healthy controls $(n=10)$. Means and standard deviations for individuals with PF and healthy controls were $15.28 \pm$ 2.76 and $18.22 \pm 4.24$ degrees, respectively. By using the $G^{*}$ Power program version 3.1.9.2 with the function of $t$-test, means: difference between two independent means and a priori chosen for power of analysis. The alpha error and power were set at 0.05 and 0.8 , respectively.

\section{Results}

\subsection{Characteristics of the participants}

Twenty-one individuals with PF and 21 healthy controls participated in the study. The means and standard deviations of age, weight, height, body mass index (BMI), clinical onset, and pain at the morning and at the worst as well as the numbers and percentages of sex, dominant side and symptom side are presented in Table 2. Comparison of the demographic data showed no differences $(p>0.05)$ in age, weight, height, sex, BMI, and side of dominant between testing groups.

Table 2. Demographic data of the participants

\begin{tabular}{|c|c|c|c|}
\hline Variables & $\begin{array}{l}\text { Individuals } \\
\text { with plantar } \\
\text { fasciitis } \\
(n=21)\end{array}$ & $\begin{array}{l}\text { Healthy } \\
\text { controls } \\
(n=21)\end{array}$ & $p$-value \\
\hline Age [years] & $53.00 \pm 9.82$ & $53.00 \pm 11.74$ & $1.000^{\mathrm{a}}$ \\
\hline Weight $[\mathrm{kg}]$ & $64.11 \pm 12.90$ & $64.04 \pm 12.12$ & $0.986^{\mathrm{a}}$ \\
\hline Height $[\mathrm{m}]$ & $160.32 \pm 8.68$ & $161.53 \pm 7.38$ & $0.629^{\mathrm{a}}$ \\
\hline Sex: Male, Female & $\begin{array}{l}8(38.10 \%), \\
13(61.90 \%)\end{array}$ & $\begin{array}{l}8(38.10 \%), \\
13(61.90 \%)\end{array}$ & $1.000^{\mathrm{b}}$ \\
\hline BMI $\left[\mathrm{kg} / \mathrm{m}^{2}\right]$ & $24.78 \pm 3.55$ & $24.45 \pm 3.65$ & $0.766^{\mathrm{a}}$ \\
\hline $\begin{array}{l}\text { Dominant side: } \\
\text { Left, Right }\end{array}$ & $\begin{array}{c}0(0 \%) \\
21(100 \%)\end{array}$ & $\begin{array}{c}1(4.76 \%), \\
21(95.24 \%)\end{array}$ & $1.000^{\mathrm{c}}$ \\
\hline $\begin{array}{l}\text { Symptom onset } \\
\text { [months] }\end{array}$ & $7.48 \pm 7.61$ & - & - \\
\hline $\begin{array}{l}\text { Symptom side: } \\
\text { Left, Right }\end{array}$ & $\begin{array}{l}7(33.33 \%), \\
14(66.67 \%)\end{array}$ & - & - \\
\hline $\begin{array}{l}\text { Morning VAS-pain } \\
\text { [scores] }\end{array}$ & $5.45 \pm 2.56$ & - & - \\
\hline $\begin{array}{l}\text { Worst VAS-pain } \\
\text { [scores] }\end{array}$ & $6.35 \pm 2.09$ & - & - \\
\hline
\end{tabular}

Data reported in mean \pm SD or $n(\%)$; BMI: Body Mass Index; VAS: Visual Analogue Scale.

${ }^{\mathrm{a}}$ Independent Sample $t$-test, ${ }^{\mathrm{b}}$ Chi-squared test, ${ }^{\mathrm{c}}$ Fisher's Exact test.

Significant difference testing at $p<0.05$.

\subsection{Patterns of the ground reaction forces (GRFs) and the multi-segment foot motion}

In Figures 1-3 show the patterns of the left and right sides as well as the symptomatic and asymptomatic sides for the GRFs and the angular displacements for the FFHF and HFTBnin healthy controls and individuals with $\mathrm{PF}$, respectively, are shown. 
The graphs showed similar patterns between healthy controls and individuals with PF groups. For the GRFs, less variability was found in healthy controls than individuals with PF. The largest variations were observed in the antero-posterior and mediolateral forces. For the multi-segment foot motions (FFHF and HFTB angles) in healthy controls, the right side showed larger amplitude motion and variation in abduction-adduction for the FFHF and in inversion-eversion for the HFTB than the left side. From these findings, the averaged left and right sides data were averaged to produce the representative data for healthy controls. However, when observing between groups, the overall variability was greater in individuals with PF, especially in the symptomatic side.
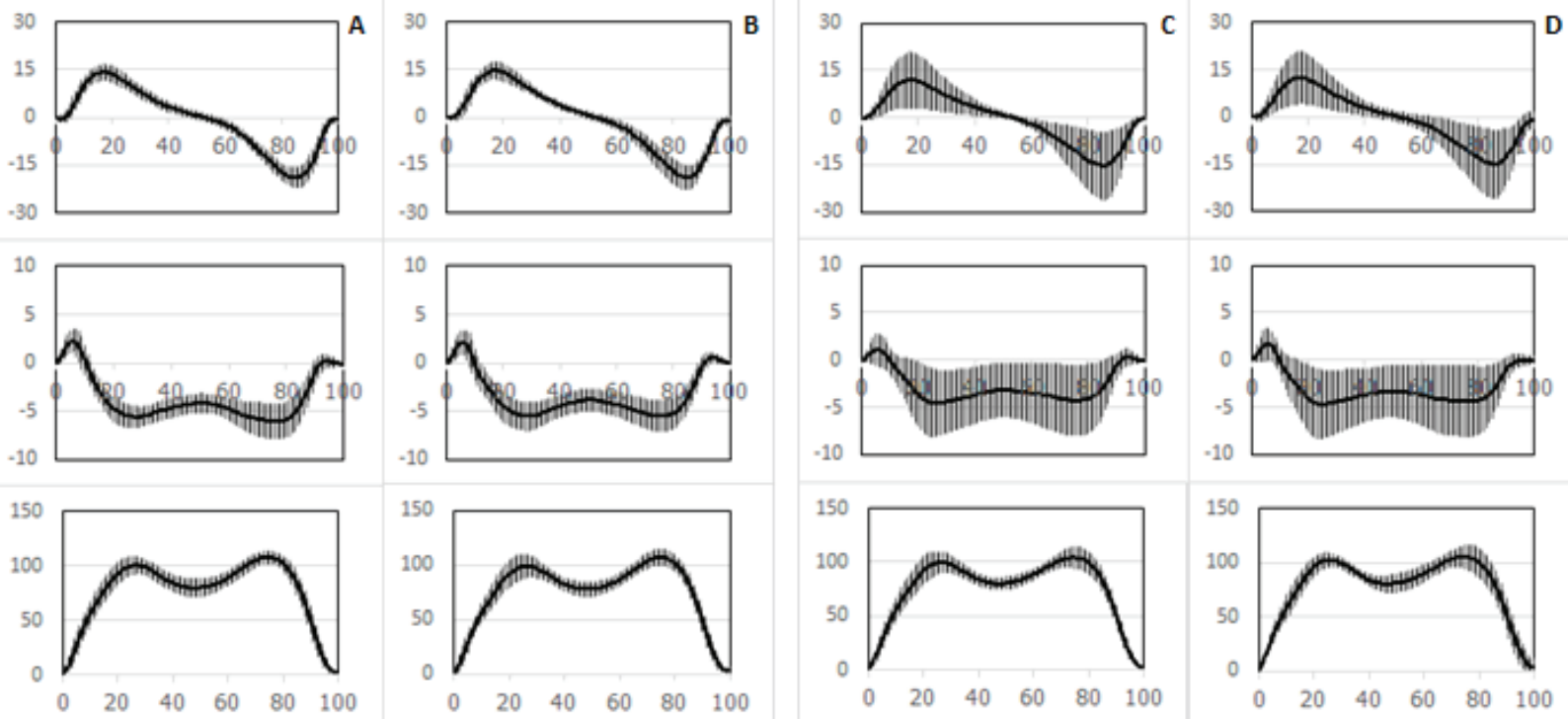

Fig. 1. Ground reaction forces (GRFs) of the healthy controls and individuals with PF,

$\mathrm{X}$-axis represents \% of gait cycle, $\mathrm{Y}$-axis represents forces in the antero-posterior (upper), medio-lateral (middle), and vertical (lower) directions; Data illustrate for the left (A) and right (B) sides of healthy controls $(n=21)$

and the symptomatic (C) and the asymptomatic (D) sides of individuals with PF $(n=21)$
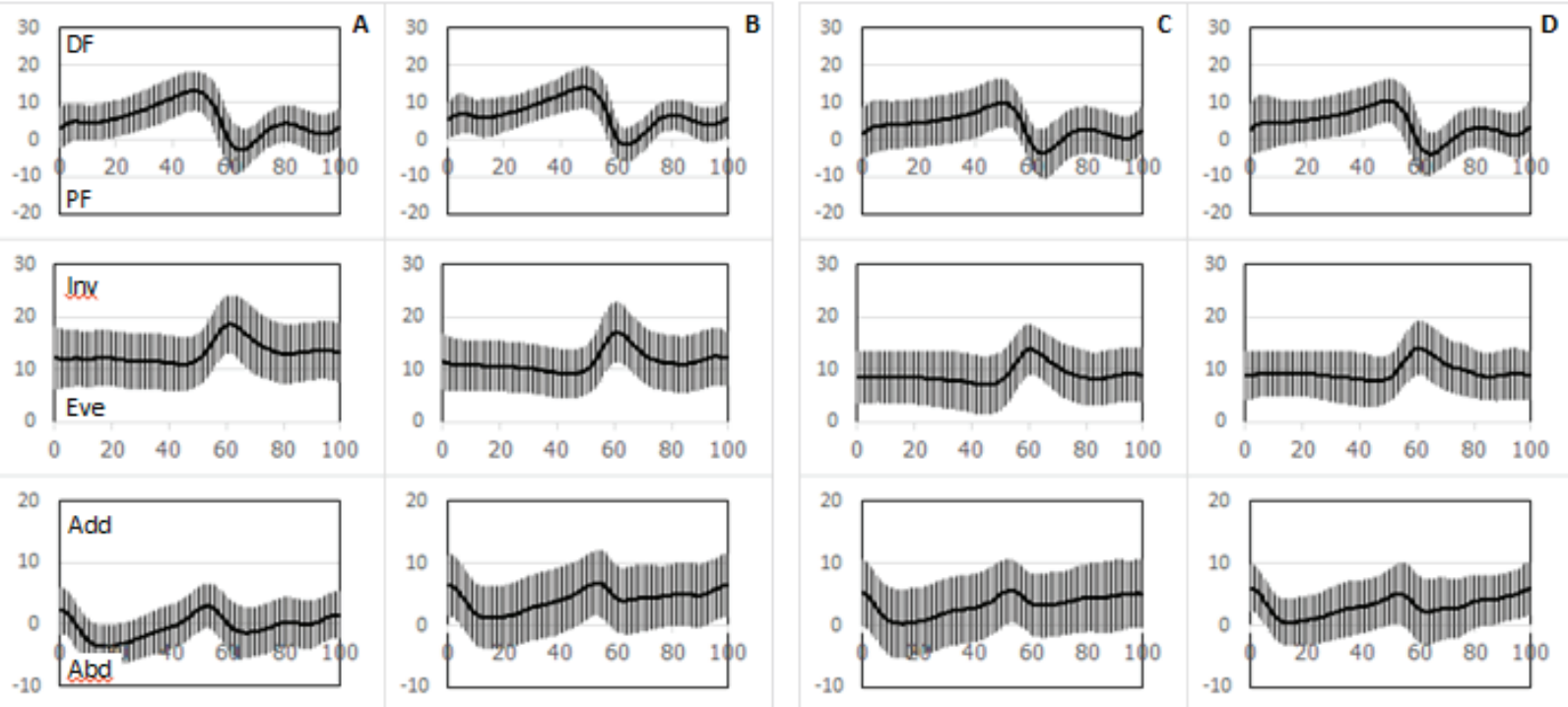

Fig. 2. The forefoot-hindfoot (FFHT) angles of the healthy controls and individuals with PF,

$\mathrm{X}$-axis represents $\%$ of gait cycle, $\mathrm{Y}$-axis represents the angles in the sagittal (upper), frontal (middle), and horizontal (lower) planes; Data illustrate for the left (A) and right (B) sides of healthy controls $(n=21)$ and the symptomatic (C) and the asymptomatic (D) sides of individuals with PF $(n=21)$ 

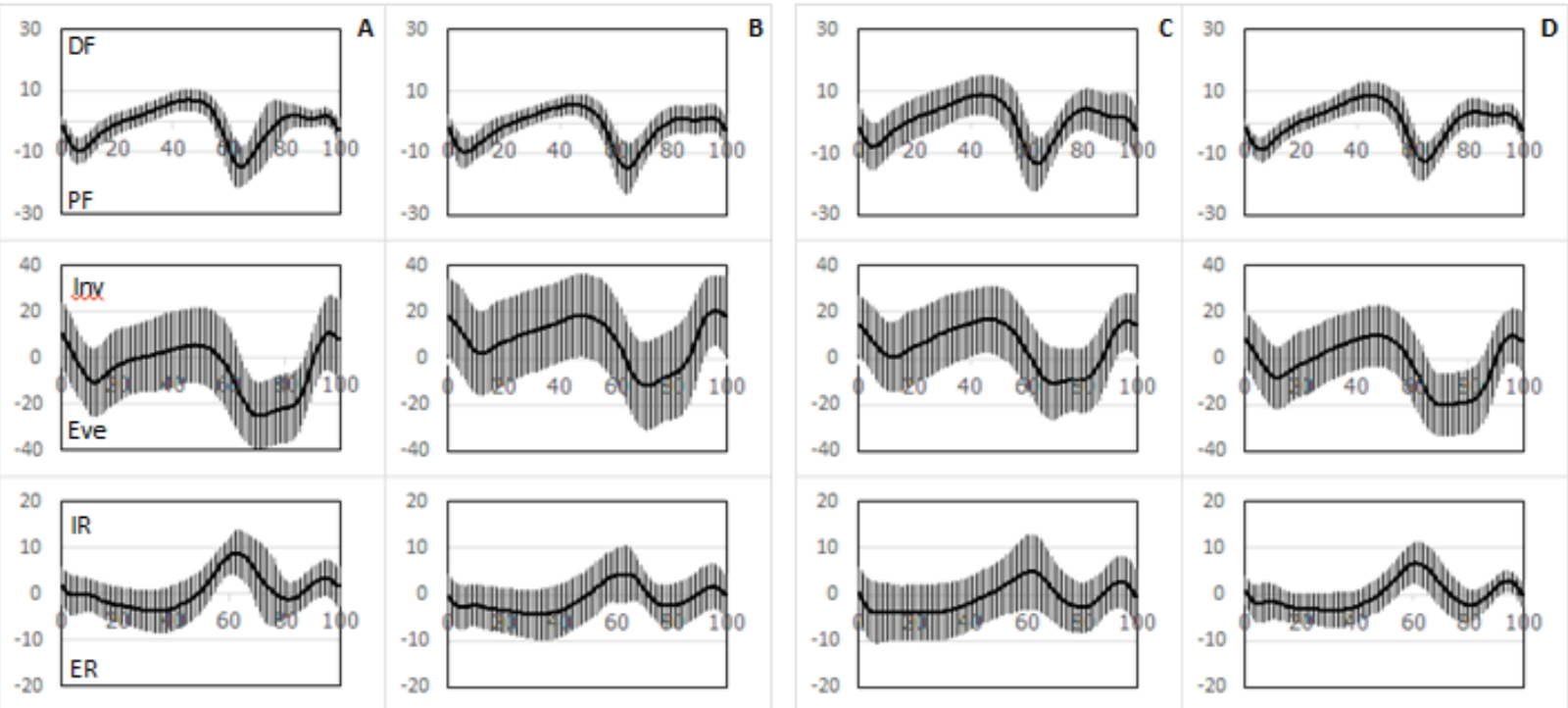

Fig. 3. The Hindfoot-tibia (HFTB) angles of the healthy controls and individuals with PF,

$\mathrm{X}$-axis represents $\%$ of gait cycle, $\mathrm{Y}$-axis represents the angles in the sagittal (upper), frontal (middle), and horizontal (lower) planes; Data illustrate for the left (A) and right (B) sides of healthy controls $(n=21)$ and the symptomatic (C) and the asymptomatic (D) sides of individuals with PF $(n=21)$

\subsection{Comparisons \\ of the temporo-spatial variables between individuals with plantar fasciitis and healthy controls}

In Table 3, comparisons of the temporo-spatial variables between individuals with PF and healthy controls are shown. There were no significant differences $(p>0.05)$ of all temporo-spatial variables between the testing groups.

\subsection{Comparisons of the ground reaction forces and multi-segment foot motions between individuals with plantar fasciitis and healthy controls}

In Table 4 comparisons of the vertical force during propulsion, breaking and propulsive forces, as well as the multi-segment foot motions in three planes of motion between the individuals with PF and healthy controls are shown. For the GRFs, there was a slight

Table 3. Comparisons of the temporo-spatial variables among the healthy controls, symptomatic, and asymptomatic sides of individuals with plantar fasciitis

\begin{tabular}{|l|c|c|c|c|c|}
\hline \multirow{2}{*}{ Variables } & \multicolumn{2}{|c|}{ Individuals with plantar fasciitis } & \multirow{2}{*}{ Healthy controls } & \multirow{2}{*}{$F$-value } & $p$-value* \\
\cline { 2 - 5 } & Symptomatic & Asymptomatic & & & 0.139 \\
\hline Cadence [steps/min] & $108.80 \pm 8.90$ & $108.84 \pm 9.39$ & $107.63 \pm 6.90$ & 0.871 \\
\hline Walking speed [m/s] & $1.05 \pm 0.11$ & $1.06 \pm 0.11$ & $1.10 \pm 0.15$ & 0.819 & 0.446 \\
\hline Stride time [s] & $1.11 \pm 0.10$ & $1.11 \pm 0.10$ & $1.12 \pm 0.07$ & 0.063 & 0.939 \\
\hline Step time [s] & $0.56 \pm 0.06$ & $0.55 \pm 0.04$ & $0.55 \pm 0.03$ & 0.102 & 0.903 \\
\hline Stride length [m] & $1.16 \pm 0.09$ & $1.18 \pm 0.10$ & $1.23 \pm 0.13$ & 1.962 & 0.149 \\
\hline Step length [m] & $0.58 \pm 0.04$ & $0.58 \pm 0.05$ & $0.60 \pm 0.06$ & 1.510 & 0.229 \\
\hline Single support time [\%GC] & $37.46 \pm 2.75$ & $37.52 \pm 2.50$ & $37.96 \pm 1.84$ & 0.279 & 0.758 \\
\hline Double support time [\%GC] & $24.22 \pm 3.90$ & $24.85 \pm 4.79$ & $23.74 \pm 3.49$ & 0.384 & 0.683 \\
\hline
\end{tabular}

* Significant difference testing by the One way ANOVA at $p<0.05$. 
reduction, but not significant for the vertical force in individuals with PF, compared to the healthy controls. The greater variability of all testing variables were found in individuals with PF, especially in the symptomatic side.

For the multi-segment foot motion, most of the variables showed lesser angles for individuals with $\mathrm{PF}$ in both the symptomatic and asymptomatic sides, compared to those of the healthy controls. Except for some variables that showed more degrees of motion but not significantly, such as the FFHF-PF, FFHF-Add, FFHF-Add/Abd-R, HFTB-DF, HFTB-Inv, and HFTB-ER angles. In individuals with $\mathrm{PF}$, there was the significant reduction of FFHF-DF angle $[\mathrm{F}(2,60)=3.141, p=0.05]$. Post-hoc analysis showed a significant difference between the symptomatic side and healthy control $(p=0.008)$. In addition, a reduction was found for the FFHF-PF/DF-R angle [F $(2,60)=3.711, p=0.03]$. Post-hoc analysis showed a significant difference between the symptomatic side and healthy control $(p=0.008)$. Significant reduction was found in FFHF-Inv angle for individuals with PF $[\mathrm{F}(2,60)=4.751$, $p=0.012]$. Post-hoc analysis showed the significant differences between the symptomatic side and healthy control $(p=0.006)$ and between the asymptomatic side and healthy control $(p=0.018)$. The data also showed significant reduction of HFTB-Inv/Eve-R $[\mathrm{F}(2,60)=3.915, p=0.025]$. Post hoc analysis showed a significant difference between the symptomatic side and healthy control $(p=0.007)$.

\section{Discussion}

The control of potential confounding factors such as age, gender, weight, and height [7] was successful as there were no differences between the two groups in the demographic data. In addition, gait may vary in accordance with the different testing environments such as surfaces or testing speed [23], [27]. Compared to walking on a treadmill, pelvic range of motion (ROM) in the frontal plane was increased but the hip ROM was decreased when walking on overground. In addition, the alteration of temporo-spatial data was found with a higher cadence when walking on a treadmill [23]. To ensure ecological validity, testing under natural walking conditions instead of using the metronome or treadmill to determine the speed was conducted. To control this aspect, we selected the trials that demon-

Table 4. Comparisons of the ground reaction forces and multi-segment foot motions between individuals with plantar fasciitis and healthy controls

\begin{tabular}{|c|c|c|c|c|c|c|c|c|}
\hline \multirow[b]{2}{*}{ Variables } & \multicolumn{2}{|c|}{ Individuals with plantar fasciitis } & \multirow{2}{*}{$\begin{array}{l}\text { Healthy } \\
\text { control }\end{array}$} & \multirow[b]{2}{*}{$F$} & \multirow[b]{2}{*}{$p$-value* } & \multicolumn{3}{|c|}{ Pairwise comparison test** } \\
\hline & Symptomatic & Asymptomatic & & & & \begin{tabular}{|l|} 
Symptomatic / \\
Asymptomatic
\end{tabular} & $\begin{array}{l}\text { Symptomatic / } \\
\text { Healthy control }\end{array}$ & $\begin{array}{l}\text { Asymptomatic/ } \\
\text { Healthy control }\end{array}$ \\
\hline Vertical force $[\mathrm{N} / \mathrm{kg}]$ & $107.01 \pm 9.27$ & $109.10 \pm 6.98$ & $109.17 \pm 5.06$ & 0.594 & 0.555 & N/A & $\mathrm{N} / \mathrm{A}$ & N/A \\
\hline Braking force $[\mathrm{N} / \mathrm{kg}]$ & $-18.25 \pm 3.92$ & $-18.18 \pm 3.83$ & $-19.26 \pm 3.16$ & 0.581 & 0.563 & $\mathrm{~N} / \mathrm{A}$ & $\mathrm{N} / \mathrm{A}$ & $\mathrm{N} / \mathrm{A}$ \\
\hline Propulsive force $[\mathrm{N} / \mathrm{kg}]$ & $15.14 \pm 3.07$ & $15.32 \pm 3.09$ & $14.93 \pm 2.61$ & 0.093 & 0.912 & $\mathrm{~N} / \mathrm{A}$ & $\mathrm{N} / \mathrm{A}$ & $\mathrm{N} / \mathrm{A}$ \\
\hline FFHF-DF [degrees] & $10.33 \pm 6.42$ & $11.46 \pm 4.98$ & $14.21 \pm 3.73$ & 3.141 & 0.050 & 0.479 & 0.018 & 0.090 \\
\hline FFHF-PF [degrees] & $-4.65 \pm 6.59$ & $-5.00 \pm 6.46$ & $-3.66 \pm 3.20$ & 0.320 & 0.727 & $\mathrm{~N} / \mathrm{A}$ & $\mathrm{N} / \mathrm{A}$ & N/A \\
\hline FFHF-DF/PF-R [degrees] & $14.98 \pm 3.21$ & $16.46 \pm 3.88$ & $17.87 \pm 3.17$ & 3.711 & 0.030 & 0.167 & 0.008 & 0.190 \\
\hline FFHF-Inv [degrees] & $14.44 \pm 4.75$ & $15.08 \pm 5.34$ & $18.81 \pm 4.78$ & 4.751 & 0.012 & 0.677 & 0.006 & 0.018 \\
\hline FFHF-Eve [degrees] & $6.33 \pm 5.18$ & $6.78 \pm 4.30$ & $9.44 \pm 4.07$ & 2.870 & 0.064 & N/A & N/A & N/A \\
\hline FFHF-Inv/Eve-R [degrees] & $8.11 \pm 2.48$ & $8.30 \pm 2.50$ & $9.37 \pm 2.25$ & 1.675 & 0.196 & $\mathrm{~N} / \mathrm{A}$ & $\mathrm{N} / \mathrm{A}$ & N/A \\
\hline FFHF-Add [degrees] & $8.14 \pm 4.76$ & $8.05 \pm 3.77$ & $6.48 \pm 3.74$ & 1.072 & 0.349 & $\mathrm{~N} / \mathrm{A}$ & $\mathrm{N} / \mathrm{A}$ & N/A \\
\hline FFHF-Abd [degrees] & $-0.79 \pm 5.11$ & $-1.08 \pm 4.26$ & $-1.84 \pm 3.18$ & 0.343 & 0.711 & $\mathrm{~N} / \mathrm{A}$ & $\mathrm{N} / \mathrm{A}$ & N/A \\
\hline FFHF-Add/Abd-R [degrees] & $8.93 \pm 2.28$ & $9.13 \pm 2.24$ & $8.33 \pm 1.77$ & 0.819 & 0.446 & $\mathrm{~N} / \mathrm{A}$ & $\mathrm{N} / \mathrm{A}$ & N/A \\
\hline HFTB-DF [degrees] & $9.54 \pm 6.58$ & $9.04 \pm 4.61$ & $6.97 \pm 2.61$ & 1.639 & 0.203 & $\mathrm{~N} / \mathrm{A}$ & N/A & N/A \\
\hline HFTB-PF [degrees] & $-14.79 \pm 8.58$ & $-14.24 \pm 5.57$ & $-17.65 \pm 6.10$ & 1.491 & 0.233 & $\mathrm{~N} / \mathrm{A}$ & N/A & N/A \\
\hline HFTB-DF/PF-R [degrees] & $24.33 \pm 5.94$ & $23.29 \pm 4.72$ & $24.63 \pm 5.20$ & 0.371 & 0.692 & $\mathrm{~N} / \mathrm{A}$ & $\mathrm{N} / \mathrm{A}$ & N/A \\
\hline HFTB-Inv [degrees] & $20.65 \pm 12.97$ & $14.36 \pm 10.88$ & $19.80 \pm 12.99$ & 1.611 & 0.208 & $\mathrm{~N} / \mathrm{A}$ & $\mathrm{N} / \mathrm{A}$ & N/A \\
\hline HFTB-Eve [degrees] & $-13.65 \pm 14.33$ & $-23.12 \pm 13.18$ & $-21.38 \pm 14.06$ & 2.775 & 0.070 & N/A & $\mathrm{N} / \mathrm{A}$ & $\mathrm{N} / \mathrm{A}$ \\
\hline HFTB-Inv/Eve-R [degrees] & $34.30 \pm 7.34$ & $37.48 \pm 7.27$ & $41.17 \pm 9.13$ & 3.919 & 0.025 & 2.000 & 0.007 & 0.138 \\
\hline HFTB-IR [degrees] & $7.00 \pm 6.90$ & $8.47 \pm 4.67$ & $9.29 \pm 4.43$ & 0.954 & 0.391 & N/A & N/A & N/A \\
\hline HFTB-ER [degrees] & $-6.26 \pm 6.21$ & $-5.50 \pm 3.26$ & $-5.34 \pm 3.90$ & 0.236 & 0.791 & $\mathrm{~N} / \mathrm{A}$ & $\mathrm{N} / \mathrm{A}$ & N/A \\
\hline HFTB-IR/ER-R [degrees] & $13.25 \pm 4.10$ & $13.97 \pm 4.18$ & $14.62 \pm 6.61$ & 0.380 & 0.685 & N/A & $\mathrm{N} / \mathrm{A}$ & N/A \\
\hline
\end{tabular}

FFHF: Forefoot with respect to hindfoot; HFTB: Hindfoot with respect to tibia; DF: Dorsiflexion; PF: Plantarflexion; Inv: Inversion; Eve: Eversion; Add: Adduction; Abd: Abduction; IR: Internal rotation; ER: External rotation; R: Range; N/A: Not assessment.

* Significant testing by the One way ANOVA at $p<0.05 ; * *$ Pairwise comparisons testing by the LSD at $p<0.05$. 
strated a similar speed between the matched-pair of participants for the analysis. Because the demographic information was not different, the natural walking speed was somewhat similar. Although the trials were selected by speed and cadence, slightly slower gait speed and shorter stride length were found in individuals with $\mathrm{PF}$, compared to the healthy controls (the difference of gait speed was $0.05 \mathrm{~m} / \mathrm{s}$ or $4.5 \%$ and the difference of stride length was $0.06 \mathrm{~m}$ or $4.8 \%$ ). However, this small difference was not likely to affect the data comparison. We compared our findings to the study of Chang et al. [4] that investigated the foot motion in individuals with PF and healthy controls, although that study tried to control the testing speed by practising until participants able to walk close to the targeted speed at $1.35 \mathrm{~m} / \mathrm{s}$. However, they still found a minimal difference in gait speed between groups at $0.03 \mathrm{~m} / \mathrm{s}$. In addition, other confounding factors such as step length and cadence, should be controlled, as suggested by the earlier studies [4], [14]. Although we did not control these factors directly, they are influenced by the personal data and, in fact, these temporo-spatial variables were not different in the present study.

Because walking is a reciprocal movement controlled by coordination between two legs, combining the data of unilateral and bilateral PF individuals may affect the results and interpretations. From the total of 45 individuals with PF who passed the criteria, only 21 with unilateral symptom were selected for the analysis in this study. Therefore, the differences found in this study provide a clearer explanation for the motion changes in individuals with PF. The participants included in this study were middle-aged to elderly and the number of women was more than men, which was consistent with the previous survey study of $\mathrm{PF}$ prevalence [16], [22]. Both groups of participants were in the normal range of BMI $(24.78 \pm 3.55$ and $24.45 \pm 3.65 \mathrm{~kg} / \mathrm{m}^{2}$ for individuals with $\mathrm{PF}$ and healthy controls, respectively). The degree of pain for individuals with PF group in the study was moderate and the onset was varied. So, data interpretation or generalizability should consider this issue.

\subsection{Ground reaction forces and the multi-segment foot motion patterns}

In individuals with $\mathrm{PF}$, the greater variability was observed in the antero-posterior and medio-latetal forces, whereas, the vertical force was more consis- tent. This may be due to the pain under the heel and fascia, causing the individuals with PF were unable to provide appropriately foot placement for the weight acceptance and push themselves in the forward progression, while the vertical force is the greatest force compared to the other two shear forces and does not change much during walking at the comfortable speed.

From the previous studies about the GRFs, conflicting findings were found [11], [13], [28]. This may be due to the difference in participant characteristics, procedure, study design, analytical process, and/or selected variables. For our results, there were no significant differences in all forces between both sides of the individuals with PF and healthy controls. This may result from the careful matching of testing speed and body weight that were implemented in this study. However, there was a tendency for adaptation in generating forces in individuals with PF. Compared to the healthy controls, the vertical force was reduced in the symptomatic side and breaking force was reduced minimally but the propulsive force was increased in both symptomatic and asymptomatic sides. This is consistent with a previous study of Liddle et al. [13], who observed no significant difference of the vertical GRF and loading rate during walking at the preferred speed between the symptomatic and asymptomatic feet in 21 individuals with unilateral PF. The authors suggested that a single factor such as the vertical GRF did not contribute to the PF alone, and other multifactorial risks should be concerned to provide a better understanding of the aetiology and management for PF. From the case series study by Hsu et al. [8], they also found no difference of the vertical GRF between symptomatic and asymptomatic sides of individuals with PF. In contrast to some of the previous studies that divided the areas under the foot into the analysis process, the study found a reduction in maximum vertical GRF at the heel and medial forefoot [26] as well as the forefoot and hindfoot [28].

For the multi-segment foot motion pattern in the healthy controls, greater variability was found in the right side. This may relate to the dominant side because it acts as a pusher during walking and facilitates forward walking progression. From the literature reviews, although gait is usually assumed to be symmetry, the subtle patterns of asymmetrical behaviour may exist with both kinematic and kinetic outcomes [21]. It was found to reflect the natural different function between lower extremities in accomplishing the tasks for propulsion and controlling when walking [21].

For individuals with $\mathrm{PF}$, the motion pattern showed greater variability especially in the symptomatic side, 
compared to the healthy controls. This variability of motion may be caused by unpredictability of the pain level. Excessive or lack of movement control can result in the negative consequences, for instance, excessive energy consumption, increased impact force, and causing an unexpected injury.

The present study found significant reductions of FFHF-DF angle about 2.75 (19.37\%) and 3.88 (27.32\%) degrees for the asymptomatic and symptomatic sides of individuals with PF when compared to the healthy controls. This may be linked to the tightness of the posterior musculotendinous structures in the leg. It was thought to be one of the major contributing factors of PF development [1], [9], [12]. Earlier pieces of evidence reported the occurrence of tightness of the posterior lower limb muscles (gastrocnemius, soleus, and hamstring) in individuals with PF [1], [9], [12]. The tightness of gastro-soleus complex would increase the tension of Achilles tendon continuing to the plantar fascia, and causing the reduced degree of ankle dorsiflexion motion [17].

In accordance with the previous results of Chang et al. [4], there was a slight increase in the FFHF-PF angle in our study. The adaptation of this part may assist the ability of forward progression of walking by producing the ankle plantarflexor force during the push-off phase. However, the present study found the FFHF-DF/PF-R angle was reduced for individuals with PF, when a previous study [4] found increasingly. For the frontal plane motion, there were significant reductions of FFHF-Inv angle about 3.73 degrees $(19.83 \%)$ and 4.37 degrees $(23.33 \%)$ in the asymptomatic and symptomatic sides, compared to the healthy controls. In addition, we found the compensatory movement with a slight increase of FFHF-Add angle, but the data showed no significance.

For the HFTB angle, a slight increase in dorsiflexion, decrease in plantarflexion, and decrease in eversion were shown, especially in the symptomatic side. In addition, there was a significant reduction of HFTB-Inv/Eve-R angle for both sides of individuals with PF. This was contrary to the previous findings [4] that found an increase of total hindfoot eversion. This may be caused by the different characteristic of the participants, testing speed, and the foot model being used.

Other factors that may affect the results of the study, such as foot pattern, plantar pressure distribution, and the tightness of posterior leg muscles should be investigated to find their effects on the multi-segment foot motion for individuals with PF in the future study. Besides the multi-segment foot motion information, as presented in this study, the intra-foot kinetic data should be ex- amined to provide more understanding about the adaptation mechanism in individuals with PF.

\section{Conclusion}

There were no significant differences in any of the GRFs between individuals with PF and healthy controls. Significant reductions were found in the FFHF-DF, FFHF-DF-R, FFHF-Inv, and HFTB-Inv/Eve-R in individuals with $\mathrm{PF}$. In addition, tendencies of the increased angles of the FFHF-PF, HFTB-DF, HFTB-Inv, and HFTB-ER, but not significant, were found in individuals with PF.

Gait analysis using the multi-segment-foot motion provides a better understanding of the adaptation in the various parts of the foot during gait in individuals with PF. Thus, the evaluation with biomechanical gait analysis together with the clinical measurements at before and after the intervention may assist the therapists to design a better treatment method for this population.

\section{Conflict of interest}

The authors declare no conflict of interest.

\section{Acknowledgements}

This research is supported by the Thailand Research Fund (TRF) Fund and Mahidol University, Thailand. We would like to thank the staffs and master students in the Physical Therapy Center, Faculty of Physical Therapy, Mahidol University, for their kindly assisted and all individuals who participated in the study.

\section{References}

[1] Bolivar Y.A., Munuera P.V., Padillo J.P., Relationship between tightness of the posterior muscles of the lower limb and plantar fasciitis, Foot Ankle Int, 2013, 34 (1), 42-48, DOI: $10.1177 / 1071100712459173$.

[2] Buchbinder R., Clinical practice. Plantar fasciitis, N. Engl. J. Med., 2004, 350(21), 2159-2166, DOI: 10.1056/NEJMcp032745.

[3] CARSOn M.C., Harrington M.E., ThOMPSON N., O'CONNOR J.J., THEOLOGIS T.N., Kinematic analysis of a multi-segment foot model for research and clinical applications: a repeatability analysis, J. Biomech., 2001, 34 (10), 1299-1307.

[4] Chang R., Rodrigues P.A., Van Emmerik R.E., Hamill J., Multi-segment foot kinematics and ground reaction forces during gait of individuals with plantar fasciitis, J. Biomech., 2014, 47 (11), 2571-2577, DOI: 10.1016/j.jbiomech.2014.06.003. 
[5] Crawford F., Thomson C., Interventions for treating plantar heel pain, Cochrane Database Syst. Rev., 2003, (3), CD000416, DOI: $10.1002 / 14651858 . C D 000416$.

[6] Dunn J.E., Link C.L., Felson D.T., Crincoli M.G., Keysor J.J., MCKINlay J.B., Prevalence of foot and ankle conditions in a multiethnic community sample of older adults, Am. J. Epidemiol., 2004, 159 (5), 491-498, DOI: 10.1093/aje/kwh071.

[7] Hernandez A., Silder A., Heiderscheit B.C., Thelen D.G., Effect of age on center of mass motion during human walking, Gait Posture, 2009, 30 (2), 217-222, DOI: 10.1016/ j.gaitpost.2009.05.006.

[8] Hsu W.H., LaI L.J., Chang H.Y., Hsu R.W., Effect of shockwave therapy on plantar fasciopathy. A biomechanical prospective, Bone Joint J., 2013, 95-B (8), 1088-1093, DOI: 10.1302/ 0301-620X.95B8.31497.

[9] IRving D.B., CoOK J.L., Menz H.B., Factors associated with chronic plantar heel pain: a systematic review, J. Sci. Med. Sport, 2006, 9 (1-2), 11-22, discussion 23-14, DOI: 10.1016/ j.jsams.2006.02.004.

[10] Irving D.B., CooK J.L., Young M.A., Menz H.B., Impact of chronic plantar heel pain on health-related quality of life, J. Am. Podiatr. Med. Assoc., 2008, 98 (4), 283-289.

[11] КатоH Y., Chao E.Y., Laughman R.K., Schneider E., MORREY B.F., Biomechanical analysis of foot function during gait and clinical applications, Clin. Orthop. Relat. Res., 1983, (177), 23-33.

[12] Labovitz J.M., YU J., KIM C., The role of hamstring tightness in plantar fasciitis, Foot Ankle Spec., 2011, 4 (3), 141-144, DOI: $10.1177 / 1938640010397341$.

[13] Liddle D., Rome K., Howe T., Vertical ground reaction forces in patients with unilateral plantar heel pain - a pilot study, Gait Posture, 2000, 11 (1), 62-66.

[14] MARTIN P.E., MARSH A.P., Step length and frequency effects on ground reaction forces during walking, J. Biomech., 1992, 25 (10), 1237-1239.

[15] Martin R.L., DAVENPORT T.E., REISChl S.F., MCPOIL T.G., MATHESON J.W., WuKICH D.K. et al., Heel pain-plantar fasciitis: revision 2014, J. Orthop. Sports Phys. Ther., 2014, 44 (11), A1-33, DOI: 10.2519/jospt.2014.0303.

[16] Nahin R.L., Prevalence and pharmaceutical treatment of plantar fasciitis in United States adults, J. Pain, 2018, 19 (8), 885-896, DOI: 10.1016/j.jpain.2018.03.003.

[17] Pascual H.J., The effect of the gastrocnemius on the plantar fascia, Foot Ankle Clin., 2014, 19 (4), 701-718, DOI: 10.1016/j.fcl.2014.08.011.

[18] Pohl M.B., Hamill J., Davis I.S., Biomechanical and anatomic factors associated with a history of plantar fasciitis in female runners, Clin. J. Sport Med., 2009, 19 (5), 372-376, DOI: $10.1097 / J S M .0 b 013 \mathrm{e} 3181 \mathrm{~b} 8 \mathrm{c} 270$.

[19] Riddle D.L., Pulisic M., Pidcoe P., Johnson R.E., Risk factors for Plantar fasciitis: a matched case-control study, J. Bone Joint Surg. Am., 2003, 85-A (5), 872-877.

[20] RidDle D.L., SCHAPPERT S.M., Volume of ambulatory care visits and patterns of care for patients diagnosed with plantar fasciitis: a national study of medical doctors, Foot Ankle Int., 2004, 25 (5), 303-310.

[21] Sadeghi H., Allard P., Prince F., Labelle H., Symmetry and limb dominance in able-bodied gait: a review, Gait Posture, 2000, 12(1), 34-45.

[22] Scher D.L., Belmont P.J., JR., Bear R., Mountcastle S.B., ORR J.D., OWENS B.D., The incidence of plantar fasciitis in the United States military, J. Bone Joint Surg. Am., 2009, 91 (12), 2867-2872, DOI: 10.2106/JBJS.I.00257.

[23] Staszkiewicz R., Chwala W., ForczeK W., Laska J., Three-dimensional analysis of the pelvic and hip mobility during gait on a treadmill and on the ground, Acta Bioeng. Biomech., 2012, 14 (2), 83-89.

[24] Stebbins J., Harrington M., Thompson N., Zavatsky A., THEOLOGIS T., Repeatability of a model for measuring multisegment foot kinematics in children, Gait Posture, 2006, 23 (4), 401-410, DOI: 10.1016/j.gaitpost.2005.03.002.

[25] Sullivan J., Burns J., Adams R., Pappas E., Crosbie J., Musculoskeletal and activity-related factors associated with plantar heel pain, Foot Ankle Int., 2015, 36 (1), 37-45, DOI: 10.1177/1071100714551021.

[26] Sullivan J., Burns J., Adams R., Pappas E., Crosbie J., Plantar heel pain and foot loading during normal walking, Gait Posture, 2015, 41 (2), 688-693, DOI: 10.1016/ j.gaitpost.2015.01.025.

[27] Sun D., Fekete G., Mei Q., Gu Y., The effect of walking speed on the foot inter-segment kinematics, ground reaction forces and lower limb joint moments, Peer J., 2018, 6, e5517, DOI: $10.7717 /$ peerj.5517.

[28] Wearing S.C., Smeathers J.E., Urry S.R., The effect of plantar fasciitis on vertical foot-ground reaction force, Clin. Orthop. Relat. Res., 2003, (409), 175-185, DOI: 10.1097/ 01.blo.0000057989.41099.d8.

[29] WINTER D.A., Biomechanics and motor control of human movement, 3rd ed., John Wiley and Sons, New Jersey, 2005.

[30] Wright C.J., Arnold B.L., Coffey T.G., Pidcoe P.E., Repeatability of the modified Oxford foot model during gait in healthy adults, Gait Posture, 2011, 33 (1), 108-112, DOI: 10.1016/j.gaitpost.2010.10.084. 